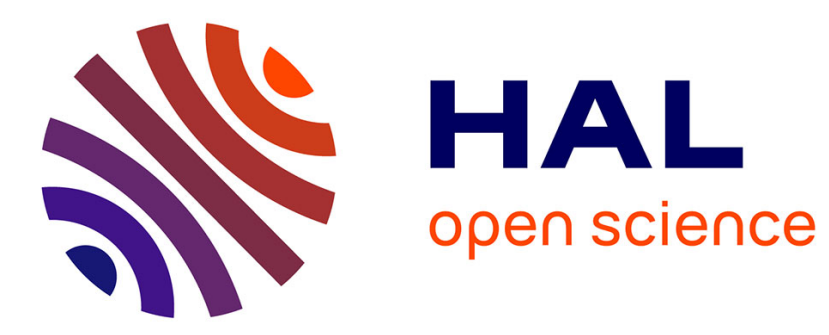

\title{
Charged Surfaces, Field Adsorption, and Appearance-Energies: an Unsolved Challenge \\ R. Forbes
}

\section{To cite this version:}

R. Forbes. Charged Surfaces, Field Adsorption, and Appearance-Energies: an Unsolved Challenge. Journal de Physique IV Proceedings, 1996, 06 (C5), pp.C5-25-C5-30. 10.1051/jp4:1996503 . jpa00254382

\section{HAL Id: jpa-00254382 https://hal.science/jpa-00254382}

Submitted on 1 Jan 1996

HAL is a multi-disciplinary open access archive for the deposit and dissemination of scientific research documents, whether they are published or not. The documents may come from teaching and research institutions in France or abroad, or from public or private research centers.
L'archive ouverte pluridisciplinaire HAL, est destinée au dépôt et à la diffusion de documents scientifiques de niveau recherche, publiés ou non, émanant des établissements d'enseignement et de recherche français ou étrangers, des laboratoires publics ou privés. 


\title{
Charged Surfaces, Field Adsorption, and Appearance-Energies: an Unsolved Challenge
}

\author{
R.G. Forbes \\ University of Surrey, Department of Electronic and Electrical Engineering, Guildford, Surrey, GU2 5XH, \\ U.K.
}

\begin{abstract}
In the context of the theory of charged surfaces, this paper re-assesses the role of field-ion appearance energy measurements in deriving information about electrical-surface position. It brings together and summarises relevant basic theory, uses existing experimental data and recent theoretical results to obtain revised numerical estimates of repulsion distance, notes some anomalies, and defines a scientific challenge.
\end{abstract}

\section{INTRODUCTION}

This paper is offered in honour of the memory of the late Professor J H Block. An important feature of his work was the development, with colleagues, especially Ernst, of field-ion appearance energy spectroscopy (FIAES) [1]. This paper looks again at how the results of FIAES may help to solve one aspect of a fundamental scientific problem. This problem is to understand, from first principles, the physics and chemistry of highly charged, atomically structured surfaces of refractory metals such as tungsten. The significance of this theoretical problem is very wide. In the context of field adsorption, Block's group and their collaborators have carried out a considerable body of work, summarised in refs. [1-4], relating to the measurement and theoretical estimation of local field values and bonding energies. This paper deals with the related issue of the electrostatic potential variation above a charged surface: in this context a prior task is to understand charged surfaces that are atomically structured but crystallographically flat. A basic concept is that of the 'electrical (reference) surface', and a central issue is its location relative to the plane of the surface atom nuclei. The paper brings together various aspects of the basic theory, and re-examines the extent to which we can derive information about electrical surface position from field-ion energy deficit measurements. It derives new numerical estimates by using recent theoretical results in re-analyses of existing experimental data, identifies some anomalies, and defines a challenge. The paper presents the main points of a longer analysis submitted for publication elsewhere [5], and is not intended as a comprehensive review. As in nearly all work on this subject, the simplifying assumption is implicitly made that data taken from real surfaces may be approximately interpreted using models for flat or atomically flat surfaces.

\section{CHARGED SURFACES: SOME BASIC CONCEPTS}

\subsection{Real charge distributions and formal excess-charge distributions}

In the discussion of charged-surface models, it is necessary to make a clear distinction between the real charge distribution (associated with the distribution of protons and electrons), and any formal excess-charge distribution (often called an 'induced charge distribution' or a 'screening charge distribution') used to represent the effects of charging the surface.

Consider a capacitor-like geometrical situation, and consider (for simplicity) that changes in the surface charge distribution are confined to a single layer of surface atoms. We charge the surface positive by removing part of the surrounding electron charge cloud from the vicinity of each surface nucleus. In reality, the resulting excess positive charge is part of the overall positive charge located at the atomic nucleus. But it is also possible to represent the change by adding to the original charge distribution a formal positive charge distribution that is everywhere equal and opposite to the removed electron charge distribution. This formal distribution is called here a formal excess-charge distribution. 
Electrostatic self-consistency requires that the removed electron charge be placed on the distant capacitor plate, where it gives rise to a uniform long-range field component $F$ ext $/ 2$. This acts to polarise the electron distribution at the positively charged surface, producing a displacement inwards of the remaining electrons. The result is a field-induced electric dipole moment associated with each surface atom. The change can be represented by the addition of a formal excess-charge distribution that is positive on the outside of the surface nuclei and negative on the inside. The two excess-charge distributions defined above add to give the total excess-charge distribution. (There is also a small displacement outwards of the surface atom nuclei, but we neglect this here.)

We thus obtain the two approaches to the representation of the charge distribution at a charged surface, one based on the real charge distribution, the other on the total excess-charge distribution. Clearly, when it comes to calculating electrostatic fields and potential outside the region occupied by the charge distribution, the same result must be obtained whichever of the two approaches is used.

\subsection{The electrical (reference) surface}

The electrical reference surface of a flat charged conductor (more usually called its 'electrical surface'), can be roughly defined as 'the plane where the field appears to start'. A more rigorous definition may be found in ref.[6]; a simplified version of this follows.

Let $Z$ be distance normal to the surface relative to an arbitrary reference zero, let the positive direction of $Z$ be outwards, let $V$ int be the classical electrostatic potential well inside the conductor, and let $F$ ext be the uniform field well above the surface. At sufficient distance from the surface (outside the range of shortrange fields associated with the structure in real surfaces), the classical electrostatic potential $V$ has the form:

$$
V-V \text { int }=-F \text { ext } \cdot\left(Z-Z_{\text {ref }}\right)=-F^{\mathrm{ext}} x
$$

The plane defined by the condition $Z=Z_{\text {ref }}$ is known as the electrical surface of the conductor. It is convenient to measure distance from the electrical surface, by defining $x=Z-Z_{\text {ref. }}$.

\section{MODELS FOR ATOMICALLY FLAT CHARGED SURFACES}

\subsection{The classical array model}

The classical array model [7] used by the present author and colleagues is based on the idea [8] that, at a charged metal surface, the individual atoms can be treated as if they behaved as polarisable dipoles. The model is a considerable over-simplification of the real situation, but provides a convenient vehicle for understanding some of the basic concepts involved. In this model the integrated excess charge associated with a particular surface atom is modelled by a (positive) point charge at the position of the surface nucleus, and the electron-polarisation excess-charge distribution by a point dipole co-located at this position. A distant array of negative charge is included for electrostatic self-consistency.

In this model, the physics of the electrical surface can be understood in the following simple manner. In the absence of the surface dipoles the electrical surface would pass through the plane of the surface nuclei [6]. The action of the surface dipoles is to try to expel the impressed field $F$ exi/ 2 due to the distant negative charge; thus the electrical surface is repelled 'outwards' from the plane of the surface nuclei (away from the interior of the conductor) by a small distance $d$. This effect has been called field repulsion; and the quantity $d$ the repulsion distance. For a given surface lattice structure there is a well-defined relationship between $d$ and the 'effective local polarisability' $b$ of a surface atom $[7,9]$. If $b$ were known independently (or could be predicted well from the free-space polarisability), then the position of the electrical surface could be predicted. Unfortunately, this has not been the case. Hist orically, the position of the electrical surface was deduced from experimental measurement of field-ion energy deficits, and the corresponding value of $b$ was then inferred via eq.(6) [9]; this value of $b$ was then used in calculations of the local field above charged surfaces [7].

Note that, since electron charge is steadily removed as the surface becomes more positively charged and the external field increases, one might expect a small decrease in the effective polarisability $b$ as the external field increases (since there is less electron charge to polarise); hence one might expect a slow movement of the electrical surface towards the plane of the surface nuclei, as external field increases. 


\subsection{Flat-surface jellium models}

In a jellium model [10] the positive ion cores are smeared out into a background charge distribution of density characterised by a parameter $r_{\mathrm{s}}$, and electrons of the corresponding number density are allowed to move against this background. The pioneering work on this method of modelling charged surfaces was done by Lang and Kohn many years ago [11], using a general linear-response theory, and there is now a considerable literature on the subject. Ref. [10] provides useful lists of references. Obviously, there is a considerable difference in the degree of sophistication of the array and jellium models, but there is also an underlying physical difference: in the array model the surface-dipole moment is laterally localised; in the jellium model (or, at least, in the simpler forms of model) there is a uniform dipole layer across the surface.

To interpret the results of jellium models, it is necessary to locate the jellium edge relative to the surface nuclei of the real surface being modelled. The conventional assumption $[10,11]$ takes the jellium edge as located at the so-called geometrical surface, which is defined to be 'outside' the surface atom nuclei by a distance equal to half the interlayer spacing normal to the surface being modelled.

A matter of considerable interest has been the effect of external-field strength on the position of the electrical surface, and it is now well established that the electrical surface (taken as the centroid of the excess-charge distribution [11]) withdraws steadily towards the jellium as the external field increases. For example, Gies and Gerhardts [12] found that, for a material with $r_{\mathrm{s}}=3 \mathrm{a}$ a. (atomic units), the centroid position moved from about $75 \mathrm{pm}$ outside the jellium edge in zero field, to about $3 \mathrm{pm}$ inside the edge at a field of $58 \mathrm{~V} / \mathrm{hm}$.

Briefly, both the array and jellium models agree that the electrical surface is outside the plane of the surface atom nuclei (i.e. that the field repulsion effect discussed earlier does occur). They also agree that the electrical surface will retreat 'inwards' as the field strength increases positively (i.e. as the surface gets more positively charged). However, they tend to disagree as to how significant this effect is: in the array model the effect looks to be small, because it depends on how much the electron removal reduces the effective local polarisability; in the jellium model the predicted effect is large. For the tungsten (111) face, where the interlayer spacing is $91 \mathrm{pm}$, the Gies and Gerhardts figures quoted above imply a reduction in repulsion distance from about $120 \mathrm{pm}$ to just over $40 \mathrm{pm}$, as the field rises from zero to $58 \mathrm{~V} / \mathrm{nm}$.

\subsection{Atomically structured flat-surface models}

In recent years, several groups (for example [13,14]) have attempted advanced self-consistent calculations that include the full three-dimensional nature of a flat atomically structured surface. The materials studied have been aluminium and silver, and the main findings are as follows:

(a) The electrical surface position is confirmed to be 'outside' the plane of the surface-atom nuclei, but tends to be closer to the surface-atom nuclei than predicted by conventional jellium models.

(b) A variation in electrical-surface position with external-field strength is found, but not as strong as in the conventional jellium models.

(c) The excess-charge distribution tends to 'pile up' on top of the surface-atom nuclei, usually outside the region occupied by the ion cores.

This 'pile-up' effect is an interesting result, but unsurprising to the present author. It confirms my view that the surface electrons respond as if they somehow belonged locally to surface atoms behaving as polarisable dipoles. The 'pile-up' finding is, of course, compatible with the well-established phenomenon of the apex location of field adsorption on relatively open crystallographic facets.

Finally, Lam and Needs [14] argue that their results suggest that the position of the electrical surface is tied not to the geometrical surface (as inherent in jellium models) but to the position of the surface atom nuclei. This, again, is a result compatible with the philosophy of the classical array model. A typical numerical result from the Lam and Needs work is as follows. For an $\mathrm{Al}(110)$ surface their calculations predict the repulsion distance as $152 \mathrm{pm}$ at zero field, reducing to $129 \mathrm{pm}$ in an external field of $30 \mathrm{~V} / \mathrm{nm}$, and to $118 \mathrm{pm}$ in a field of $45 \mathrm{~V} / \mathrm{nm}$ (if the certainty of prior field evaporation is ignored).

\section{ELECTRICAL SURFACE POSITION AND FIELD ION ENERGY DEFICITS}

There has been strong interaction between basic theory and the experimental FIAES work of Block's group. This section re-assesses progress, which has not been entirely straightforward. Refs. $[1,15]$ provide background for those not familiar with this topic. A crucial experimental fact is that electron-stimulated field desorption (ionization) (ESFD) of field-adsorbed atoms and normal field ionization (FI) of imaging-gas atoms can take place together, and both sets of ion energies can be measured. 


\subsection{Previous work}

A parameter of interest is the difference $\Delta D_{1}$ between the ion energies corresponding to the onset of the FI distribution and the peak of the ESFD distribution. For uniform surface field $F$, we have [16]:

$\Delta D_{1}=\left(I_{1}-\phi \mathrm{E}+\eta_{1}^{\mathrm{b}}-U_{0^{\mathrm{c}}}\right)-e F x^{\mathrm{b}}$,

where $I_{1}$ is the gas-atom ionization energy, $\phi_{\mathrm{E}}$ is the local emitter work-function, $\eta 1^{\mathrm{b}}$ is the 'purely chemical' interaction between the surface and the newly created ion, $U_{0} \mathrm{c}$ is the potential energy of the neutral at the critical surface, and $e$ is the elementary positive charge. $x^{\mathrm{b}}$ denotes the field-adsorption bonding distance, i.e. the distance of the nucleus of the firmly field-adsorbed atom from the electrical surface. If no term in the bracket varies significantly with field, then $\Delta D_{1}$ will be a linearly decreasing function of field. Note that the bonding distance $x^{b}$ differs from the tungsten-helium bond length $z^{\mathrm{b}}$, which is the distance between the two nuclei. The two parameters are related via the repulsion distance $d$ defined earlier, by:

$x^{\mathrm{b}}=z^{\mathrm{b}}-d$.

The first relevant experiments were made by Culbertson et al. [16] with a magnetic sector analyser, which measures $\Delta D_{1}$ directly. For the $\mathrm{He}$ on $\mathrm{W}$ system, straight-line results were obtained. Culbertson et al. thus derived values of $x^{\mathrm{b}}$ from the gradients of their plots, obtaining values between $164 \mathrm{pm}$ and $180 \mathrm{pm}$. Subsequently [9], the present author used these data and eq.(3) to make estimates of repulsion distance. With a value $259 \mathrm{pm}$ for the tungsten-helium bond length, as used earlier by Tsong and Müller [17], results for $d$ were between $79 \mathrm{pm}$ and $95 \mathrm{pm}$. To the best of my knowledge, this was the first empirically derived estimate of the real physical position of the electrical surface.

\subsection{The work of Block's group and collaborators}

Some years later, Kreuzer and Nath developed new calculations on field adsorption [18], using the socalled ASED-MO method. These produced values for tungsten-helium bond length $z^{\mathrm{b}}$ somewhat different from the $259 \mathrm{pm}$ assumed by Tsong and Müller, and also suggested field-dependence in bond length.

At the same time, new measurements of field-ion energy deficits were being carried out by Ernst and coworkers [19], and a new method of analysing these measurements was developed [15,19]. This was based, not on relative measurement of the two lines (values of $\Delta D_{1}$ derived from Ernst's work are much the same as those of Culbertson et al.), but on absolute measurements of the appearance energies for the ESFD ions from the field adsorbed layer. Details are in ref. [15], and a slightly generalised theory in ref. [5]. Assuming a uniform surface field, and using a 'reduced' image potential to represent the ion-surface interaction, Ernst converted measured energy deficits into distances $x$ from the electrical surface. Hence the ESFD distribution peak can be used to give the helium-tungsten bonding distance $x^{b}$, and field-dependent peak shifts can be converted to a variation of $x^{b}$ with field. Fig. 1 shows the resulting data-points.

Fig. 1. Bonding distance measured from the electrical surface, as a function of external field, for helium field-adsorbed on tungsten. Plotted points represent the experimentallybased results of Ernst et al. [19], and the horizontal line the experimental results of Culbertson et al. [16]. The curve $E$ is derived from the cluster calculations of Kreuzer and Nath [18], with no local-field effects taken into account, and is fitted as described in the text. The filled triangle is the reference result, taken here to be a bonding distance of $131 \mathrm{pm}$ at a external field of $38 \mathrm{~V} / \mathrm{nm}$.

Curves A and B show theoretical estimates derived from LDF calculations [4]. Curve A: with local-field effects disregarded. Curve B: with field enhancement by a factor of 1.5 .

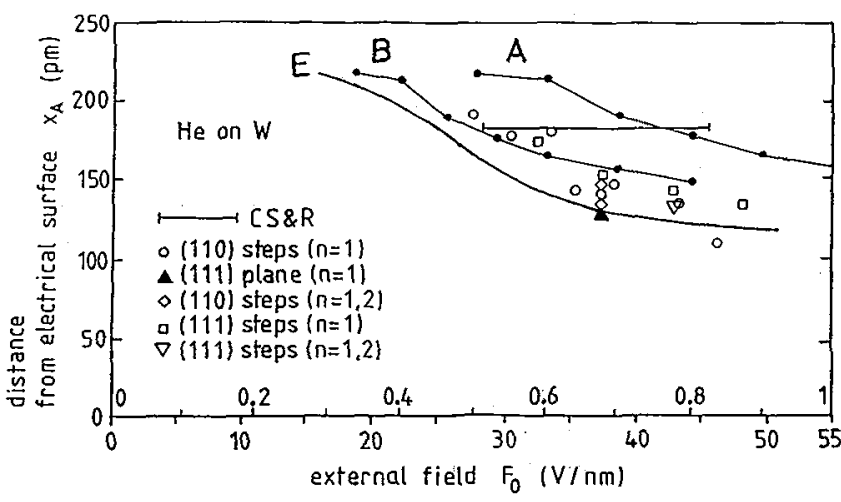


These experimental results for $x^{\mathrm{b}}$ exhibit a field dependence broadly similar to the Kreuzer and Nath theoretical results for $z^{\mathbf{b}}$. Thus, after using a scaling procedure to deal with a discrepancy in the evaporation field predicted by the ref. [18] calculations, Ernst et al. [19] were able to compare the two sets of results, and to use eq.(3) to obtain a repulsion-distance $(d)$ estimate of $80 \mathrm{pm}$. A data point for the (111) plane, at an external-field value of $38 \mathrm{~V} / \mathrm{nm}$, was used as the reference result for this comparison; this procedure leads to curve E in Fig. 1. Later, ref. [15] used a slightly different method, based on combining energy deficit values for singly and doubly-charged ESFD ions; analysis leads to a reference result [15] here taken as $x^{\mathrm{b}}=133 \mathrm{pm}$ at external field $43 \mathrm{~V} / \mathrm{nm}$, and to a $d$-estimate [15] of $73 \mathrm{pm}$. Perhaps somewhat by chance, these estimates of $d$ were similar to those originally derived from the work of Culbertson et al. (79 pm to $95 \mathrm{pm}$ ). So, as noted in ref.[6], there seemed at the time to be no wider theoretical implications.

But actually there is a self-consistency problem. The theoretical curve in Fig. 2 of ref. [18] suggests that in the external-field range used by Culbertson et al. ( $30 \mathrm{~V} / \mathrm{nm}$ to $45 \mathrm{~V} / \mathrm{nm}$ ), bond length varies from about $240 \mathrm{pm}$ to $200 \mathrm{pm}$. The average value over the range is perhaps $210 \mathrm{pm}$. A bond length of $210 \mathrm{pm}$ applied to the Culbertson et al. results for $x^{\mathrm{b}}$ would imply estimates of $d$ in the range $25 \mathrm{pm}$ to $40 \mathrm{pm}$. Such distances seem somewhat low, and are not consistent with the results derived by the other methods. These low distances raise doubts, therefore, either about one or more methods of data analysis, or about the quantitative results of the theoretical bond-length calculations, or about the scaling procedure.

Recently, another new theoretical approach to field adsorption has been developed, by Wang [4]. This uses a jellium model and employs local density functional (LDF) formalism. After a small correction [4] for field-dependence in electrical-surface position relative to the jellium edge, curve A in Fig.1 is obtained.

However, in the LDF calculations on bonding energies [4], in order to get a good match between theoretical and experimental values, it was necessary to use a model-field value significantly higher, by a factor approaching 1.5, than the external-field value (i.e. the 'measured field') associated with the relevant experimental observations. For consistency, this philosophy has to be carried over into the bondingdistance comparisons. As shown in Fig. 1, the agreement between (a) the empirical data of Ernst et al. for a given external-field value, and (b) the theoretical data for a model field equal to this external field (curve A) is not particularly good. But when theoretical results for a model field higher than the external field by a factor of 1.5 are used, curve $B$ in Fig. 1 results and the agreement is better.

Unfortunately, scrutiny of the ref. [19] procedure for experimental data analysis uncovers a difficulty. The field value $F$ used in that analysis clearly should be an average field between the electrical surface and the field adsorption site. But if it is important to get this field right in the theoretical calculations, then we also have to get it right in the data analysis. In which case, for a given external field $F$ ext, it is required that the data analysis should set $F$ equal to (in this case) $1.5 F$ ext, rather than equal to $F$ ext. If this is done, then the apparent good fit between curve B and the experimentally-derived data-points is removed, because the data points derived by the method described in ref. [19] move to smaller values of $x^{b}$.

The philosophy of 'using the local field' also needs to be applied to the numerical estimations performed earlier. In the Culbertson et al. approach, if we take the local field between the electrical surface and the bonding site to be 1.5 times the external field, then values of bonding distance $x^{\mathrm{b}}$ derived from their experiments are less by this factor, and lie between $110 \mathrm{pm}$ and $120 \mathrm{pm}$. In the Ernst et al. [19] approach, using the same factor of 1.5, the reference result in Fig. 1 now corresponds to an average local field of about $57 \mathrm{~V} / \mathrm{nm}$, and recalculation of $x^{\mathrm{b}}$ gives a value of about $80 \mathrm{pm}$. However, a new anomaly appears, because the ref. [15] method that combines deficits for singly and doubly charged ions is not dependent on assumed field value; so the result $133 \mathrm{pm}$ is unchanged (but now corresponds to a local field of $64 \mathrm{~V} / \mathrm{nm}$ ).

To obtain revised estimates of repulsion distance we need relevant theoretical estimates of tungsten-helium bond-length. For model fields near $60 \mathrm{~V} / \mathrm{nm}$, my best estimate from existing calculations $[4,18,19]$ is $210 \pm 20 \mathrm{pm}$. This gives repulsion-distance estimates lying (for the three approaches) either near $90 \mathrm{pm}$ or near $130 \mathrm{pm}$ or near $80 \mathrm{pm}$. It is unfortunate that the various approaches currently give results that differ significantly, but perhaps encouraging that including local-field effects (where relevant) gives estimates closer to the results of the recent quantum-mechanical calculations for aluminium and silver.

\section{DISCUSSION AND CONCLUSIONS}

I draw several conclusions from this brief re-examination. First, with the advent of advanced selfconsistent quantum-mechanical calculations, it is interesting that in some ways the physical picture emerging from these seems close to the intuitive scientific perceptions developed by the field-ion microscopists. 
In the derivation of bonding distances, both the theoretical (LDF) calculations and the later experimentallyderived results show the same qualitative trend of a field-related reduction in bonding distance. However, there are significant, systematic, differences between the theoretical and empirical results, and between empirical results derived in different ways. These discrepancies remain to be explained.

It has also become abundantly clear that a good estimate of the repulsion distance (i.e. a good empirical estimate of the location of the electrical surface) requires accurate knowledge both of the bonding distance $x^{b}$ and of the field-adsorption bond length $z^{\mathrm{b}}$. Only the quantity $x^{\mathrm{b}}$ can be derived from energy-deficit measurements (and this not without uncertainty). If the charged surface in a field-adsorption calculation is modelled in atomic detail, giving a prediction of $z^{\mathrm{b}}$, then we can put this modelling together with energydeficit data to produce a repulsion-distance estimate. But if the charged surface is modelled by structureless jellium, then our deductions about bond length and repulsion distance will be constrained by the uncertainties associated with the use of jellium models in such a context.

The discrepancies between the various empirical approaches, and unanticipated problems that limit accurate independent prediction of field-adsorption bond length and its variation with field, are bad news, because at present they significantly limit the accuracy with which we can empirically determine the repulsion distance. On the other hand, the perception that FIAES can give us information about the adsorbate interaction with the substrate immediately after ionization is encouraging: for if we can eventually establish by good theoretical modelling what the purely electrostatic effects are, then FIAES measurements should be able to provide useful information about ion-surface interactions at a charged surface.

Almost certainly, we have reached (more likely, well passed!) the limit of what can be usefully done with simple uniform-field models. There is a now an urgent need for more precise modelling of threedimensional details, even for crystallographically flat surfaces. This enhanced modelling is needed, first for the prediction of how fields and potentials vary in space (especially the location of the electrical surface), second for the prediction of field-adsorption bond lengths and bonding energies, and third for the investigation of the 'purely chemical' interactions between an external ion and a charged surface.

Clearly, the real need is for better quantum-mechanical treatments of charged atomically-structured surfaces, as I have long advocated [7]. The development of an embedded cluster model is useful [20]. However, advanced self-consistent computational techniques do now exist, and have been applied to aluminium and silver. For the field-ion emission community, there is an urgent need to now get these modelling techniques applied to materials, such as tungsten, for which energy-deficit measurements exist. In return, one can argue that these experimental results from the fundamental FIAES work on field adsorption provide an extra area - and potentially a very fruitful area - in which the physical predictions of such quantum-mechanical calculations can be tested and validated against experiment. The challenge proposed by this paper is to carry through such a scientific program.

\section{REFERENCES}

[1] Ernst N., Appl. Surface Sci. 67 (1993) 82-96.

[2] Kreuzer H.J. and Wang R.L.C., Phil. Mag. 69 (1994) 945-955.

[3] Suchorski Yu., Schmidt W.A., Ernst N., Block J.H. and Kreuzer H.J., Prog. Surf. Sci. 48 (1995) 121.

[4] Wang R.L.C., Kreuzer H.J. and Forbes R.G., Surface Sci. 350 (1996) 183-205.

[5] Forbes R.G., Z. Angw. Phys. (Neue Folge) (submitted for publication).

[6] Forbes R.G., Surface Sci. 223 (1989) 326-352.

[7] Forbes R.G. and Wafi M.K., Surface Sci. 93 (1980) 192-212.

[8] Drechsler M., Z. Electrochem. 61 (1957) 48.

[9] Forbes R.G., Appl. Phys. Letters 36 (1980) 739-740.

[10] Kiejna A. and Wojciechowski K.F., Metal Surface Electron Physics (Pergamon, Oxford, 1996).

[11] Lang N.D. and Kohn W., Phys. Rev. B 7 (1973) 3541-3550.

[12] Gies P. and Gerhardts R.R., Phys. Rev. 10A (1986) 982-989.

[13] Inglesfield J.E., Phil. Trans. Roy. Soc. Lond. A 334 (1991) 527-538.

[14] Lam S.C. and Needs R.J., J. Phys.: Condens. Matter 5 (1993) 2101-2108.

[15] Ernst N., Surface Sci. 219 (1989) 1-32.

[16] Culbertson R., Sakurai T. and Robertson G.H., Phys. Rev. B 19 (1979) 4427.

[17] Tsong T.T. and Müller E.W., J. Chem. Phys. 55 (1971) 2884-2889.

[18] Kreuzer H.J. and Nath K., Surface Sci. 183 (1987) 591-608.

[19] Ernst N., Drachsel W., Li Y., Block J.H., and Kreuzer H.J., Phys. Rev. Letters. 57 (1986) 2686.

[20] Wang R.L.C. and Kreuzer H.J., Surface Sci. 323 (1995) 333-339. 